\title{
Are women living with HIV prone to osteoporosis in postmenopause? A systematic review
}

\author{
Pérsio Yvon Adri Cezarino', MD \\ Ricardo dos Santos Simões, MD', PhD \\ Edmund Chadat Baracat, MD', PhD \\ José Maria Soares Junior, MD', PhD
}

1. Discipline of Gynecology, Department of Obstetrics and Gynecology, Hospital das Clínicas, Faculty of Medicine,

University of São Paulo (HC-FMSP), São Paulo, SP, Brazil

http://dx.doi.org/10.1590/1806-9282.64.05.469

\begin{abstract}
SUMMARY
BACKGROUND: Some researchers have suggested that HIV infections can increase the cytokines, which might interfere with the bone metabolism and increase the risk of bone mass loss. However, this issue has yet to be consolidated in postmenopausal women.

OBJECTIVE: To analyze studies that evaluated the loss of bone mass through DEXA in women living with HIV.

MATERIALS AND METHODS: A systematic review was conducted following the PRISMA guideline. The MEDLINE, EMBASE and Cochrane databases were consulted from January 1987 to March 2017. Studies assessing bone mineral density (BMD) in postmenopausal women living with HIV were included. The secondary outcome was to evaluate the impact of antiretroviral on BMD.

RESULTS: Sixty percent of the manuscripts suggested that women living with HIV had more bone loss than women in the control group, mainly in the lumbar spine. Forty percent did not observe any difference between groups. One study reported the influence of antiretroviral drugs on bone mass but did not find any difference between groups.

CONCLUSION: Our data suggest that HIV infections may have a negative influence on bone mass loss in women. Further studies on the mechanism of this HIV consequence are necessary to clarify the connection as well as the impact of the antiretroviral action on BMD in postmenopausal women.
\end{abstract}

KEYWORDS: Climacteric. Osteoporosis. HIV. Bone Density.

\section{INTRODUCTION}

HIV infections are related to systemic inflammatory processes, which may increase some mediators and cytokines that play a role in the regulation of several endocrine and metabolic processes. In fact, HIV infections can increase the TNF-alpha e IL-6, thus increasing bone resorption., ${ }^{1,2}$ Therefore, information on the bone metabolism of patients infected with the human immunodeficiency virus (HIV) is of real interest due to the regularly recurring changes of physiologic states during the course of disease. ${ }^{3,4}$
A clinical trial with 112 HIV-infected men reported that users of protease inhibitors presented a 2.2fold enhanced relative risk of bone loss (osteopenia or osteoporosis) on the whole-body measurement ${ }^{4}$. Notwithstanding, the majority of studies focused only on a male young-adult population around peak bone mass, for which osteoporosis prevalence is not high $^{5}$. However, it is not clear if the loss of body mass in female patients is only related to HIV infections or the used drugs. ${ }^{5-8}$ Therefore, the aim of this research was to analyze studies and evaluate the influence of HIV on the women bone mass as a first outcome.

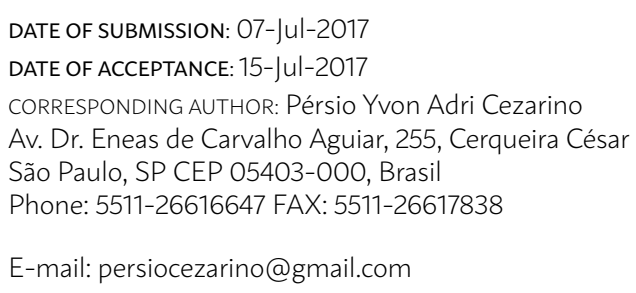
ecbaracat@gmail.com 


\section{METHODOLOGY}

This systematic review of the literature followed the PRISMA (Preferred Reporting Items for Systematic Reviews and Meta-Analysis) guidelines ${ }^{9}$. The databases used for consultation were MEDLINE and Cochrane. Articles published in English, Spanish, Portuguese, Italian and French from 1987 (the first study of HIV) to September 2016 were selected using the search strategies reported in Fig. 1. Systematic reviews, meta-analyses, and references cited in the papers were consulted, but only original work was included in this review. The study selection, as well as the evaluation of titles and abstracts, was carried out by two researchers (P.Y.A.C. and R.S.S.), skilled in conducting blind systematic reviews independently, who closely followed the inclusion and exclusion criteria. After this stage, the original articles were critically assessed before being included or not in the study. When selection became a source of discord, a third reviewer was consulted (J.M.S.).

In the inclusion of studies, preference was shown for prospective observational studies or clinical trials. Furthermore, they should include postmenopausal women living with HIV who were undergoing antiretroviral therapy and were informed about bone mineral density. The latter was measured using dual-energy X-ray absorptiometry (DEXA). Also, based on the WHO criteria for the osteoporosis diagnosis, a T-score below or equal to -2.5 SD (standard deviation) for lumbar spine, femoral neck, and/or total femur was adopted. $^{10}$

The eligibility criteria for the studies in this review consisted of women between 45 and 65 years of age, who were in postmenopause, and who had received antiretroviral therapy for more than five years. Studies of populations with characteristics different from those just mentioned were excluded.

When the evidence selected after applying the inclusion criteria was defined as a randomized controlled clinical trial, it was run through an appropriate checklist for critical evaluation (JADAD). Studies were considered for inclusion if the JADAD score was equal to or higher than $3 .^{11}$ When the evidence was defined as a comparative study (observational studies or nonrandomized clinical trials), it was also run through an appropriate checklist for critical evaluation (NEWCASTLE OTTAWA), and only those studies with a score over 6 were included.12.

The information gleaned from the selected studies was displayed in a table comprising the following variables: authors' names, publication year, study design, number of patients, CD4 count, age, form and duration of antiretroviral therapy, duration of HIV infection, and expected outcome (Table 1). To date,

TABLE 1 - THE CHARACTERISTICS AND PRIMARY OUTCOME OF SELECTED STUDIES.

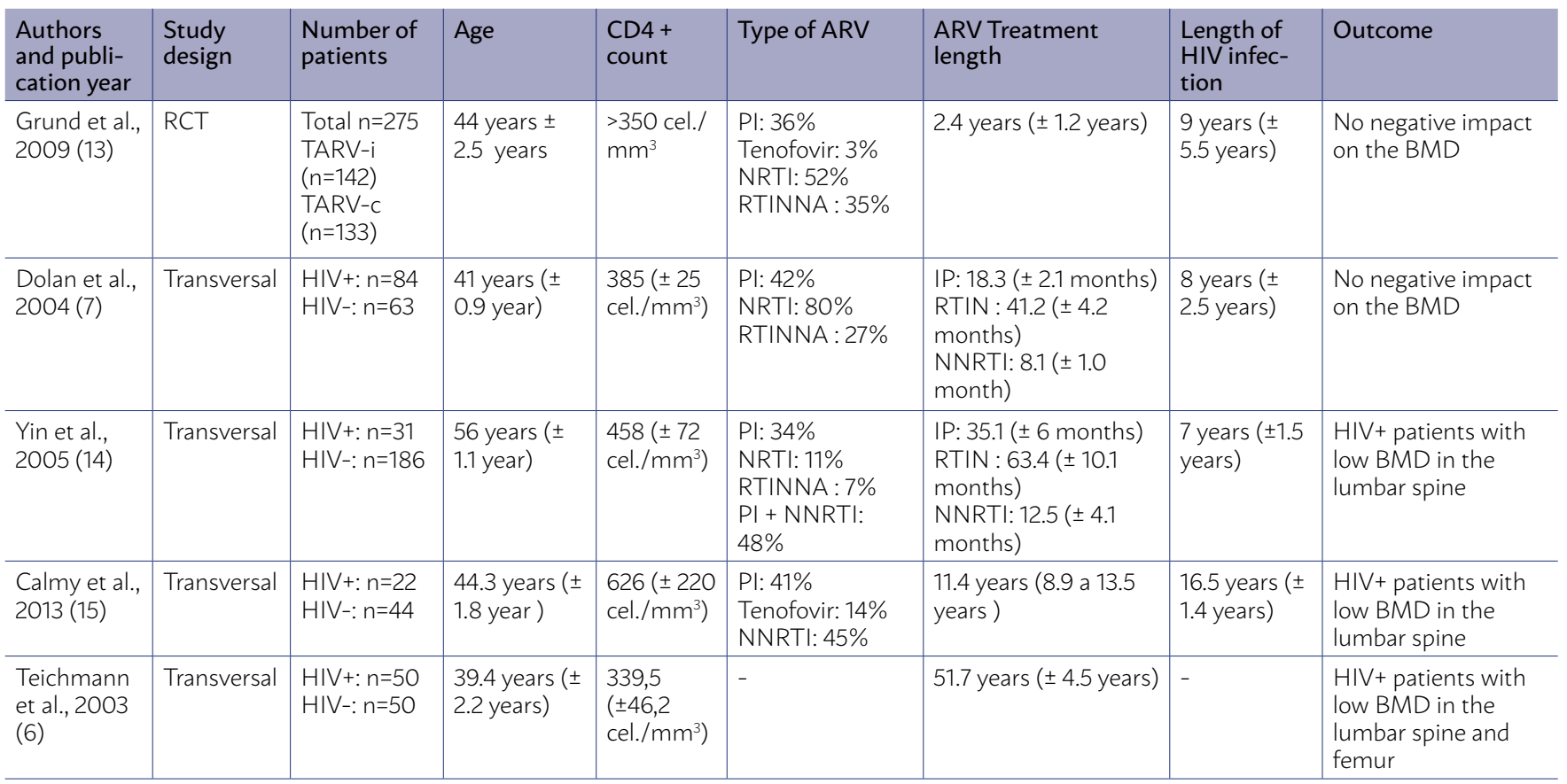

$\mathrm{RCT}=$ randomized clinical trial; $\mathrm{N}$ = number; ARV: antiretroviral; IP: protease inhibitor; $\mathrm{RTIN}$ = reverse transcriptase inhibitor nucleoside analogue; RTINNA = reverse transcriptase inhibitors, non-nucleoside analogues; BMD = Bone Mass Density 
Medline: (Highly Active Antiretroviral Therapy OR HAART OR Antiretroviral Therapy, Highly Active OR Anti-HIV Agents OR Agents, Anti-HIV OR Anti HIV Agents OR Anti-AIDS Agents OR Agents, Anti-AIDS OR Anti AIDS Agents OR Anti-HIV Drugs OR Anti HIV Drugs OR Drugs, Anti-HIV OR AIDS Drugs OR Drugs, AIDS OR Anti-AIDS Drugs OR Anti AIDS Drugs OR Drugs, Anti-AIDS OR CCR5 Receptor Antagonists OR HIV Fusion Inhibitors OR HIV Integrase Inhibitors OR HIV Protease Inhibitors) AND (Osteoporosis OR Osteoporosis, Post-Traumatic OR Osteoporosis, Post Traumatic OR Post-Traumatic OR Osteoporosis OR Post-Traumatic Osteoporosis OR Osteoporosis, Senile or Osteoporosis, Senile OR Senile Osteoporosis OR Senile Osteoporosis OR Osteoporosis, Involutional OR Osteoporosis, Age-Related OR Osteoporosis, Age Related and Bone Loss, Age-Related and OR Age-Related Bone Loss OR Age-Related Bone Losses OR Bone Loss, Age Related OR Bone Losses, Age-Related OR Age-Related Osteoporosis OR Age Related Osteoporosis OR Age-Related Osteoporosis OR Osteoporosis, Age-Related)

Cochrane: Highly Active Antiretroviral Therapy AND Osteoporosis

FIG. 1 - Databases and search strategies.

there is not a sufficient number of randomized clinical trials to perform a meta-analysis, which would provide a more robust analysis.

\section{RESULTS}

A total of 347 articles were identified in the primary database. After titles and abstracts were carefully read and the inclusion criteria were applied, five studies were selected for critical evaluation (Fig. 2). Table 1 presents their main characteristics. There were altogether 462 women who were living with HIV and whose median age was 44 years \pm 6.5 years. The mean length of HIV infection was $8.1 \pm 3.2$ years and the CD4 cell count varied from 339 to 626 (482.5 \pm 71.5 cells). The mean length for ART treatment was $6.3 \pm 1.2$ years; only the randomized clinical trial conducted by Grund et $\mathrm{al}^{13}$ was included. Also, there is not a sufficient number of randomized clinical trials to perform a meta-analysis.

Sixty percent of manuscripts suggested that women living with HIV had more bone loss than women in the control group, especially for lumbar spine ( $\mathrm{n}=$ 409 patients with HIV) ${ }^{13-15}$. Forty percent did not observe any difference between groups. One study reported the influence of antiretroviral drugs on bone mass but did not find any difference between groups (Table 1). In fact, the study using continuous ART or with CD4 count inferior to 250 cells/mm3 was not related to osteoporosis at the lumbar spine (RRA=0,012 com 95\% CI: -0.053 a 0.042) ${ }^{13}$.

\section{DISCUSSION}

Retroviral therapy has brought a new perspective to women living with HIV and prolongs the longevity, thus increasing the number of women entering the menopause stage, when there is a reduction in the production of steroids. ${ }^{16-20}$ However, there are questions on whether women have a higher propensity to osteoporosis due to HIV infection and the effect of ARVs hypoestrogenism during this period. Our study suggests that HIV negatively influences bone mass density in postmenopausal women. ${ }^{6,14,16}$ However, we found no evidence that ARVs could increase this risk, because of a low number of studies.

Women infected by HIV may be asymptomatic for 10 years, as the reduction in lymphocytes is slow. ${ }^{21}$ However, the infection can determine a loss of bone mass due to a decrease in the health of the patient and changes in the immune system ${ }^{21}$. Indeed, increased levels of cytokines such as TNF present in chronic HIV infection can increase bone turnover by stimulation of osteoclasts ${ }^{17,18}$ and enhancement in bone resorption. In more severe forms of infection by HIV, low levels of CD4 are also related to reduced bone mineral density, as demonstrated in the study cohort. ${ }^{19}$ In these cases, opportunistic infections, a decrease in nutrition, reduced bone mass and general condition could be the factors that negatively influence bone mass. ${ }^{16-19}$

Three studies evaluated the risk of osteoporosis in HIV+ population. ${ }^{6,14,16}$ However, only the study of Teichmann et al. ${ }^{6}$ found an increase in osteoporosis risk for HAART users. The authors did not report the time of HIV infection, previous use of antiretroviral therapy prior to the study, or the ART composition used. That is a concern regarding this study. Another limitation of the studies was the absence of factor makers, which can make data interpretation more difficult. Also, It is known that type of drug used in HAART is essential, because it may be responsible for a higher or lower impact in the bone microenvironment. For example, reverse transcriptase inhibitors (NRTIs) and protease inhibitors (PI) act in blocking RANKL decreased calcitriol and inhibition in osteoblasts. In turn, Zidovudine accelerates the genesis of osteoclasts and Tenofovir impairs bone 


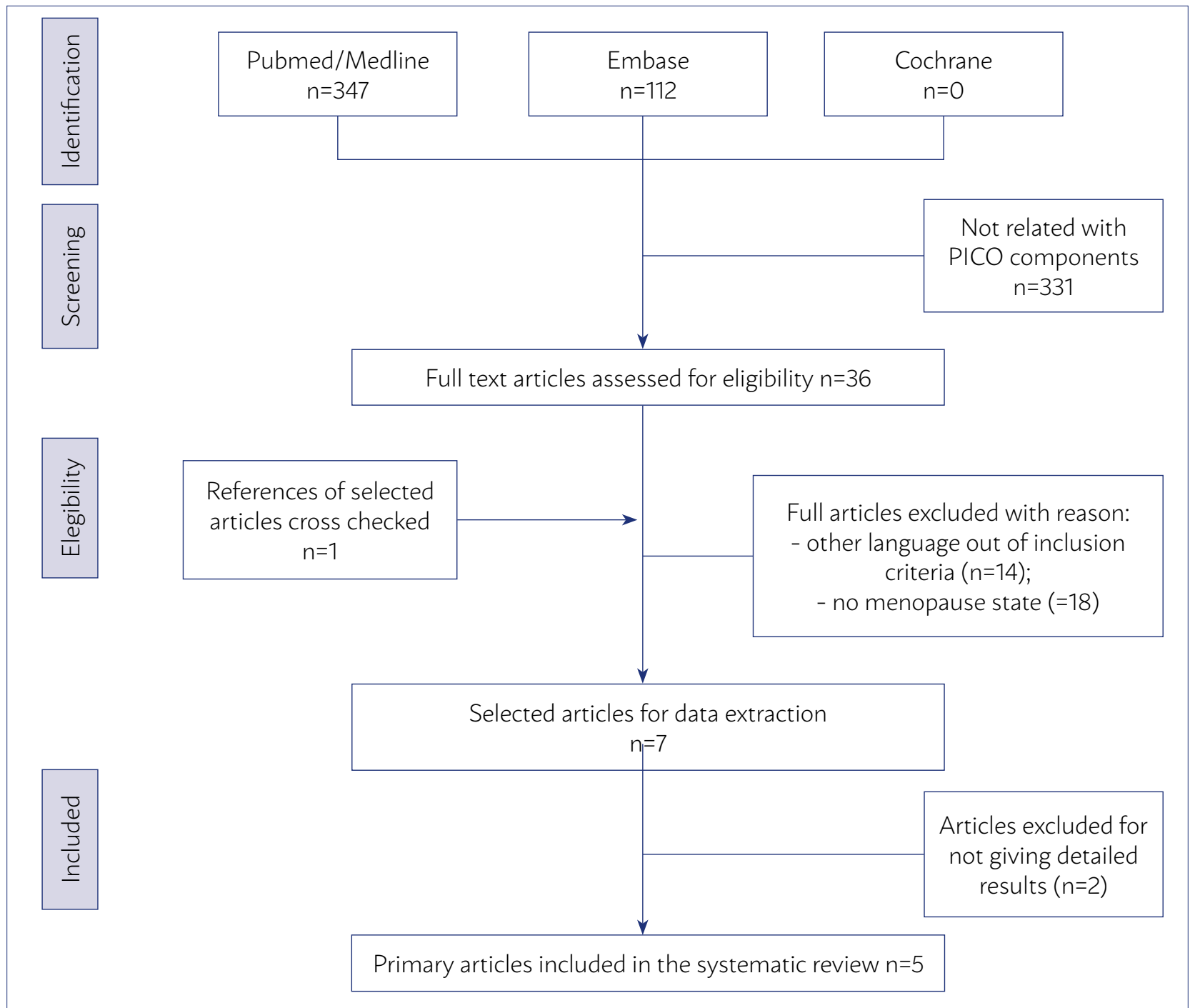

FIG. 2 - Systematic review algorithm.

mineralization. Thus, the composition is essential to evaluate the use of HAART in bone mass and risk of osteoporosis, which can be a limitation of the studies analyzed.

Dolan et al. ${ }^{7}$ reported the type of ART used and time of HIV infection but failed to inform HAART use time before inclusion in the study. Additionally, there was heterogeneity in the population, including women who were still menstruating and with higher levels of estrogen, which complicates the assessment of osteoporosis related to low estrogen levels. Furthermore, there is a selection bias in study type, primary outcome, and population in the selected randomized studies, ${ }^{13}$ which may hinder the risk analysis of osteoporosis in women with HIV during the climacteric. Also, another limitation of our study was the lack of a sufficient number of randomized clinical trials to perform a meta-analysis, which would provide a more robust analysis. Another bias was the study of
Teichmann et al. ${ }^{6}$ related to the inclusion of young patients.

The survival of women living with HIV has dramatically increased because of retroviral drugs ${ }^{15}$. However, there is concern that these substances have an adverse impact on bone mass, mainly in climacteric women, who experience the effects of hypoestrogenism on the bone density. ${ }^{11}$ Therefore, there is great concern about the bone mass loss in the HIV population.

\section{CONCLUSION}

Finally, our data suggest that HIV infections may have a negative influence on the bone mass loss in postmenopausal women. Further studies on the mechanism of this HIV consequence are necessary to clarify this connection as well as the impact of the antiretroviral action on BMD in postmenopausal women. 


\section{REFERENCES}

1. Cao W, Mehraj V, Kaufmann DE, Li T, Routy JP. Elevation and persistence of CD8 T-cells in HIV infection: the Achilles heel in the ART era. J Int AIDS Soc. 2016;19(1):20697.

2. Serrano-Villar S, Sainz T, Lee SA, Hunt PW, Sinclair E, Shacklett BL, et al. HIV-infected individuals with low CD4/CD8 ratio despite effective antiretroviral therapy exhibit altered $T$ cell subsets, heightened CD8+ T cell activation, and increased risk of non-AIDS morbidity and mortality. PLoS Pathog. 2014;10(5):e1004078.

3. Brown TT, Qaqish RB. Antiretroviral therapy and the prevalence of osteopenia and osteoporosis: a meta-analytic review. AIDS. 2006;20(17):2165-74.

4. Tebas P, Powderly WG, Claxton S, Marin D, Tantisiriwat W, Teitelbaum $\mathrm{SL}$, et al. Accelerated bone mineral loss in HIV-infected patients receiving potent antiretroviral therapy. AIDS. 2000;14(4):F63-7.

5. Amiel C, Ostertag A, Slama L, Baudoin C, N'Guyen T, Lajeunie E, et al. BMD is reduced in HIV-infected men irrespective of treatment. J Bone Miner Res. 2004;19(3):402-9.

6. Teichmann J, Stephan E, Lange U, Discher T, Friese G, Lohmeyer J, et al. Osteopenia in HIV-infected women prior to highly active antiretroviral therapy. J Infect. 2003;46(4):221-7.

7. Dolan SE, Huang JS, Killilea KM, Sullivan MP, Aliabadi N, Grinspoon S. Reduced bone density in HIV-infected women. AIDS. 2004;18(3):475-83.

8. Bolland MJ, Grey AB, Gamble GD, Reid IR. CLINICAL Review \#: low body weight mediates the relationship between HIV infection and low bone mineral density: a meta-analysis. J Clin Endocrinol Metab. 2007;92(12):4522-8.

9. Moher D, Liberati A, Tetzlaff |, Altman DG; PRISMA Group. Preferred reporting items for systematic reviews and meta-analyses: the PRISMA statement. Ann Intern Med. 2009;151(4):264-9, W64.

10. Assessment of fracture risk and its application to screening for postmenopausal osteoporosis. Report of a WHO Study Group. World Health Organ Tech Rep Ser. 1994;843:1-129.

11. Jadad AR, Moore RA, Carroll D, Jenkinson C, Reynolds DJ, Gavaghan DJ, et al. Assessing the quality of reports of randomized clinical trials: is blinding necessary? Control Clin Trials. 1996;17(1):1-12
12. Wells GA, Shea B, O'Connell D, Peterson J, Welch V, Losos M, et al. The Newcastle-Ottawa Scale (NOS) for assessing the quality of nonrandomised studies in meta-analyses. [cited 2017 Jun 2]. Available from: http://www.ohri.ca/programs/clinical_epidemiology/oxford.asp

13. Grund B, Peng G, Gibert CL, Hoy JF, Isaksson RL, Shlay JC, et al; INSIGHT SMART Body Composition Substudy Group. Continuous antiretroviral therapy decreases bone mineral density. AIDS. 2009;23(12):1519-29.

14. Yin M, Dobkin J, Brudney K, Becker C, Zadel JL, Manandhar M, et al. Bone mass and mineral metabolism in HIV+ postmenopausal women. Osteoporos Int. 2005;16(11):1345-52

15. Calmy A, Chevalley T, Delhumeau C, Toutous-Trellu L, Spycher-Elbes R, Ratib $\mathrm{O}$, et al. Long-term HIV infection and antiretroviral therapy are associated with bone microstructure alterations in premenopausal women. Osteoporos Int. 2013;24(6):1843-52.

16. Arora S, Agrawal M, Sun L, Duffoo F, Zaidi M, lqbal ). HIV and bone loss. Curr Osteoporos Rep. 2010;8(4):219-26.

17. Aukrust P, Haug C), Ueland T, Lien E, Müller F, Espevik T, et al. Decreased bone formative and enhanced resorptive markers in human immunodeficiency virus infection: indication of normalization of the bone-remodeling process during highly active antiretroviral therapy. I Clin Endocrinol Metab. 1999;84(1):145-50.

18. Ueland T, Frøland SS, Bollerslev J, Aukrust P. Increased levels of biochemical markers of bone turnover in relation to persistent immune activation in common variable immunodeficiency. Eur J Clin Invest. 2001;31(1):72-8.

19. Cazanave C, Dupon M, Lavignolle-Aurillac V, Barthe N, Lawson-Ayayi S, Mehsen N, et al; Groupe d'Epidémiologie Clinique du SIDA en Aquitaine. Reduced bone mineral density in HIV-infected patients: prevalence and associated factors. AIDS. 2008;22(3):395-402.

20. Fonseca AM, Bagnoli VR, Arie WMY, Azevedo Neto RS, Couto Jr. EB, Baracat EC. Dados demográficos, epidemiológicos e clínicos de mulheres brasileiras climatéricas. São Paulo: Casa Leitura Médica; 2010. p.23-251.

21. Carvalho EH, Gelenske T, Bandeira F, Albuquerque MF. Bone mineral density in HIV-infected women taking antiretroviral therapy: a systematic review. Arq Bras Endocrinol Metabol. 2010;54(2):133-42. 